\title{
Training to use EUS-FNA : It is time to give up the human hands-on approach?
}

Authors

Institutions
Marc Barthet ${ }^{1}$, Geoffroy Vanbiervliet ${ }^{2}$, Jean-Michel Gonzalez ${ }^{1}$

Service de Gastroentérologie, Hôpital NORD AP-HM, Aix-Marseille-Université, Marseille, France

'CHU l'Archet 2, Nice, France submitted:

1. February 2016

accepted after revision:

8. February 2016

\section{Bibliography}

DOI http://dx.doi.org/

10.1055/s-0042-102957

Published online: 11.8 .2016

Endoscopy International Open

2016; 04: E1009-E1010

(c) Georg Thieme Verlag KG

Stuttgart $\cdot$ New York

E-ISSN 2196-9736

\section{Corresponding author}

Georffroy Vanbiervliet

Service de Gastroentérologie Hôpital NORD AP-HM

Aix-Marseille-Université

Marseille

France

vanbiervliet.g@chu-nice.fr
In this issue, Hoshi $\mathrm{K}$ et al evaluate their endoscopic ultrasound fine-needle aspiration (EUSFNA) training model [1]. This model involves use of an isolated porcine stomach and is believed to be realistic, simple, and inexpensive. Training in EUS, and mainly in EUS-FNA, is really challenging because use of the procedure is growing and it is key to medical management, particularly in oncology. EUS-FNA requires expertise and practioners need to be trained to perform the procedure. More than 10 years ago, the American Society for Gastrointestinal Endoscopy determined that achieving competency in EUS procedures required a minimum of 150 supervised cases, at least 50 of which were EUS-FNA [2]. Other recent recommendations have stated a threshold of 75 EUS-FNA cases [2]. Results of a follow-up study have shown that a special training program could significantly reduce the median number of passes after 100 EUS procedures and the morbidity after 200 procedures [3]. Few training centers offer minimal training during a 1-year curriculum. Over the past 10 years, our team has developed a special nationwide learning and training program for EUS and EUS-FNA that includes 2 weeks of theoretical courses involving video libraries and 1 week of hands-on training involving procedures on live pigs [4]. We have shown an increase in competency with diagnostic EUS and EUS-FNA at the end of this course [4].

If training is required and helpful for accurate diagnosis and skill in performing EUS-FNA, the problem is where, when, and how to obtain that training. As previously noted, training centers are scarce, with approximately 25 in the United States and one to three in each European country $[2,4]$. However, at more and more workshops and digestive disease meetings, an EUS-FNA teaching platform is being offered to preregistered candidates that includes tabletop isolated porcine models. These courses are not widespread, however, and regular training is required.
When to do EUS-training is another question. Training in EUS-FNA typically is reserved for physicians who have completed residency or are seeking postgraduate credentials because it requires both cognitive and technical competencies and because EUS scopes are not available in all endoscopy units. Finally, the cornerstone for EUS and EUS-FNA training is the method by which the skills are gained. A training model for EUSFNA should be realistic, that is, the image and scope positioning should be consistent. The model should also be easy to reproduce and the training widely available and inexpensive so that expertise can be diffused. Durability, too, is important, meaning that the model holds up through many procedures done by inexperienced hands in a training session of at least a half day.

Many forms of training in EUS-FNA have been proposed for young practioners, including didactic materials with video libraries, a computerbased virtual model, tabletop phantom models, ex vivo models that use isolated organs from animals, and finally live pigs. From an anatomical perspective in comparison with human beings, training to perform US on live pigs is the most exciting and realistic way to learn. However, it is also the most expensive and the model to which it is the most difficult to gain access because of restrictive laws and the costs involved (the price of the animal, the veterinarian, the animal laboratory, etc.) $[2,4]$.

Fritsher-Ravens et al recently demonstrated that porcine lymph nodes in live pigs are a good model for acquiring EUS-FNA competence [5]. Models with isolated animal organs may be the best compromise in terms of realism, cost, and availability. The organs most often used are pig stomach to simulate false submucosal tumors, such as in the paper by Hoshi et al. that appears in this issue (chicken tenderloins), sutured colon segments filled with various fluid contents to simulate pseudocystic lesions, or grapes to mimick lymph 
nodes [6]. Isolated organs can be surrounded with water, tapioca, agar gel or gelatin to enhance the accuracy of US. Based on current practice with US and during repeated procedures by inexperienced hands, however, these models could be more or less rapidly damaged or destroyed. Therefore, achieving realistic properties for and durability of models for EUS-FNA training remains challenging. Many teams are working to develop a realistic, efficient, durable, and cheap model for teaching EUS-FNA. Guidelines from the European Society of Gastrointestinal Endoscopy recommend a combination of different simulators and, if possible, a live swine model to achieve competency in performing EUS-FNA [7].

The model developed by Hoshi's team is a new contribution for improving training in EUS-FNA [1]. In order for skills in EUS-FNA to be diffused, training models that are cheap, realistic, and efficient are required because achieving proficiency with the procedure requires skills and a long learning curve with a minimum threshold, which is difficult to attain and to validate [8]. But the availability of that kind of model needs to be increased and it should be validated in comparative studies and its real impact on the quality of EUS-FNA assessed. Therefore, it is definitely time to give up human hands-on training in EUS-FNA.

\section{References}

1 Hoshi K, Irisawa A, Shibukawa G et al. Realistic, simple and inexpensive EUS-FNA training model produced from isolated porcine stomach: feasibility and repeatability testing. EIO 2016: DOI 10.1055/s-0042110094

2 Paquin S. Training in endsocopic ultrasound-guided fine needle aspiration. Endosc Ultrasound 2014; 3: $12-16$

3 Eloubeidi MA, Tamhane A. EUS-guided FNA of solid pancreatic masses : a learning curve with 300 consecutive procedures. Gastrointest Endosc 2005; 61: 700-708

4 Barthet M, Gasmi M, Boustiere C et al. EUS training in live pig model: does it improve echoendoscope hands-on and trainee competence? Endoscopy 2007; 39: 535-539

5 Fritsher-Ravens A, Cuming T, Dhar $S$ et al. EUS-FNA training. Evaluation of a new porcine lymphadeiopathy models for in vivo hands-on teaching and training, and review of the literature. Endoscopy 2013; 45: $114-120$

6 Baron TH, DeSimio TM. New ex-vivo porcine model for endoscopic ultrasound-guided training in transmural puncture and drainage of pancreatic cysts and fluid collections. Endosc Ultrasound 2015; 4: 34-39

7 Polkowski M, Larghi A, Weynand B et al. Learning, techniques, and complications of EUS-sampling in gastroenterology: ESGE technical guideline. Endoscopy 2012; 44: 190-205

8 Wani S, Coté GA, Reswani R et al. Learning curves for EUS by using cumulative sum analysis : Implications for American Society for Gastrointestinal Endoscopy recommendations for training. Gastrointest Endosc 2013; 77: 558-565 